\title{
Discretization of the Poisson equation with non-smooth data and emphasis on non-convex domains*
}

\author{
Thomas Apel ${ }^{\dagger} \quad$ Serge Nicaise Johannes Pfefferer $^{\ddagger}$
}

May 7, 2015

\begin{abstract}
Several approaches are discussed how to understand the solution of the Dirichlet problem for the Poisson equation when the Dirichlet data are non-smooth such as if they are in $L^{2}$ only. For the method of transposition (sometimes called very weak formulation) three spaces for the test functions are considered, and a regularity result is proved. An approach of Berggren is recovered as the method of transposition with the second variant of test functions. A further concept is the regularization of the boundary data combined with the weak solution of the regularized problem. The effect of the regularization error is studied.

The regularization approach is the simplest to discretize. The discretization error is estimated for a sequence of quasi-uniform meshes. Since this approach turns out to be equivalent to Berggren's discretization his error estimates are rendered more precisely. Numerical tests show that the error estimates are sharp, in particular that the order becomes arbitrarily small when the maximal interior angle of the domain tends to $2 \pi$.
\end{abstract}

Key Words Elliptic boundary value problem, method of transposition, very weak formulation, finite element method, discretization error estimate

AMS subject classification $65 \mathrm{~N} 30 ; 65 \mathrm{~N} 15$

\footnotetext{
${ }^{*}$ The work was partially supported by Deutsche Forschungsgemeinschaft, priority program 1253 and IGDK 1754.

${ }^{\dagger}$ thomas.apel@unibw.de, Universität der Bundeswehr München, Institut für Mathematik und Bauinformatik, D-85579 Neubiberg, Germany

${ }^{\ddagger}$ snicaise@univ-valenciennes.fr, LAMAV, Institut des Sciences et Techniques de Valenciennes, Université de Valenciennes et du Hainaut Cambrésis, B.P. 311, 59313 Valenciennes Cedex, France

$\S$ johannes.pfefferer@unibw.de, Universität der Bundeswehr München, Institut für Mathematik und Bauinformatik, D-85579 Neubiberg, Germany
} 


\section{Introduction}

The motivation for this paper is to consider the boundary value problem

$$
-\Delta y=f \quad \text { in } \Omega, \quad y=u \quad \text { on } \Gamma:=\partial \Omega,
$$

with right hand side $f \in H^{-1}(\Omega)$ and boundary data $u \in L^{2}(\Gamma)$. We assume $\Omega \subset \mathbb{R}^{2}$ to be a bounded polygonal domain with boundary $\Gamma$. Such problems arise in optimal control when the Dirichlet boundary control is considered in $L^{2}(\Gamma)$ only, see for example the papers by Deckelnick, Günther, and Hinze, [11], French and King, [12], May, Rannacher, and Vexler, [17], and Apel, Mateos, Pfefferer, and Rösch, [1]. On the continuous level we even admit more irregular data.

In Section 2 we analyze several ways how to understand the solution of the boundary value problem (1.1) for which we cannot expect a weak solution $y \in H^{1}(\Omega)$. The most popular method to solve problem (1.1) is the transposition method that goes back to Lions and Magenes [16] and that is based on the use of some integration by parts. This formally leads to the very weak formulation: Find $y \in Y$ such that

$$
(y, \Delta v)_{\Omega}=\left(u, \partial_{n} v\right)_{\Gamma}-(f, v)_{\Omega} \quad \forall v \in V
$$

with $(w, v)_{G}:=\int_{G} w v$ denoting the $L^{2}(G)$ scalar product or an appropriate duality product. The main issue is to find the appropriate trial space $Y$ and test space $V$. In the convex case, it turns out that a good choice is $Y=L^{2}(\Omega)$ and $V=H^{2}(\Omega) \cap H_{0}^{1}(\Omega)$. This case is investigated by many authors, some of them are mentioned in Subsection 2.1 , but we will see that such a choice is not appropriate in the non-convex case (loss of uniqueness) and present some remedies (enlarged test spaces) in Subsections 2.2 and 2.3. We will further show in Subsection 2.2 that in the correct setting the very weak solution corresponds to the one obtained by an integral equation technique and hence has $H^{1 / 2}(\Omega)$ regularity.

The main drawback of the very weak formulation is the fact that a conforming discretization of the test space should be made by $C^{1}$-elements. Hence Berggren proposed in [4] to introduce two new variables, $\varphi:=-\Delta v$ and $\zeta:=\partial_{n} v$ allowing to perform a simpler numerical analysis, see Subsection 2.4. In Subsection 2.5 we propose another method that consists of regularizing the Dirichlet datum $u$ by approximating it by a sequence of functions $u^{h}$ in $H^{1 / 2}(\Gamma)$ using for example an interpolation operator. This allows to compute a sequence of weak solutions $y^{h} \in H^{1}(\Omega)$, and we show that they converge to the very weak solution with an explicit convergence rate.

A negative result about the well-posedness of the weak formulation with $L^{1}(\Gamma)$-data completes the discussion on the continuous level.

Section 3 is devoted to the numerical analysis. We start with Berggren's numerical approach and recall his error estimates in Subsection 3.1 for completeness. Next we perform in Subsection 3.2 a numerical analysis of our regularization approach and prove error estimates for the piecewise linear approximation on a family of conforming, quasi-uniform finite element meshes. Notice that it turns out that on the discrete level Berggren's approach is a particular case of our regularization strategy. The convergence 
order is $\frac{1}{2}$ in the convex case but smaller in the non-convex case. This reduction can be explained by the singular behaviour of the solution of the dual problem. In our paper [2] we investigate the singular complement method to remedy the suboptimality of the standard finite element method in non-convex domains.

Finally, in Section 4 we present numerical tests in order to illustrate that our error estimates are sharp. The paper ends with some remarks about the three-dimensional case and about data with different regularity than assumed above.

\section{Analysis of the boundary value problem}

In this section we analyze several ways how to understand the solution of the boundary value problem (1.1) for which we cannot expect a weak solution $y \in H^{1}(\Omega)$.

For keeping the notation succinct we assume that the polygonal domain $\Omega$ has at most one non-convex corner with interior angle, denoted by $\omega$. Let $r, \theta$ be the corresponding polar coordinates and define $\lambda:=\pi / \omega$. The boundary segments of $\Omega$ are denoted by $\Gamma_{j}$, $j=1, \ldots, N$.

\subsection{Method of transposition in convex polygonal domains}

The method of transposition goes back at least to Lions and Magenes, [16], and is used by several other authors including French and King, [12], Casas and Raymond, [6], Deckelnick, Günther, and Hinze, [11], and May, Rannacher, and Vexler, [17]. Since by partial integration the derivation

$$
\begin{aligned}
(f, v)_{\Omega} & =-(\Delta y, v)_{\Omega}=(\nabla y, \nabla v)_{\Omega} & & \text { for } v \in H_{0}^{1}(\Omega) \\
& =\left(y, \partial_{n} v\right)_{\Gamma}-(y, \Delta v)_{\Omega} & & \text { for } v \in H^{2}(\Omega) \cap H_{0}^{1}(\Omega)
\end{aligned}
$$

is valid we get the very weak formulation: Find

$$
y \in L^{2}(\Omega): \quad(y, \Delta v)_{\Omega}=\left(u, \partial_{n} v\right)_{\Gamma}-(f, v)_{\Omega} \quad \forall v \in H^{2}(\Omega) \cap H_{0}^{1}(\Omega) .
$$

May, Rannacher, and Vexler, [17], proved the existence of a solution in $L^{2}(\Omega)$ in the case of a convex polygonal domain $\Omega$.

Lemma 2.1. If the domain $\Omega$ is convex there exists a unique solution $y \in L^{2}(\Omega)$ of problem (2.1) that satisfies the a priori error estimate

$$
\|y\|_{L^{2}(\Omega)} \leq c\left(\|u\|_{\prod_{j=1}^{N} H_{00}^{1 / 2}\left(\Gamma_{j}\right)^{\prime}}+\|f\|_{\left(H^{2}(\Omega) \cap H_{0}^{1}(\Omega)\right)^{\prime}}\right)
$$

provided that $u \in \prod_{j=1}^{N} H_{00}^{1 / 2}\left(\Gamma_{j}\right)^{\prime}$ and $f \in\left(H^{2}(\Omega) \cap H_{0}^{1}(\Omega)\right)^{\prime}$.

Recall that $H_{00}^{1 / 2}\left(\Gamma_{j}\right)$ is the space of functions whose extension by zero to $\Gamma$ is in $H^{1 / 2}(\Gamma)$. 
Proof. For being self-contained we sketch here the proof of [17, Lemma 2.1]. The idea is first to assume more regular data, $u \in H^{1 / 2}(\Gamma), f \in H^{-1}(\Omega)$ such that a weak solution $y \in H^{1}(\Omega)$ exists, then to show (2.2), and finally to use a standard density argument since $H^{2}(\Omega) \cap H_{0}^{1}(\Omega) \stackrel{c}{\hookrightarrow} H_{0}^{1}(\Omega)$ and $H^{1 / 2}(\Gamma) \stackrel{c}{\hookrightarrow} L^{2}(\Gamma)=\prod_{j=1}^{N} L^{2}\left(\Gamma_{j}\right) \stackrel{c}{\hookrightarrow} \prod_{j=1}^{N} H_{00}^{1 / 2}\left(\Gamma_{j}\right)^{\prime}$.

The estimate (2.2) is proven by using a duality argument. Due to the convexity of the domain there exists a solution $w \in H^{2}(\Omega)$ of the auxiliary problem

$$
-\Delta w=y \quad \text { in } \Omega, \quad w=0 \quad \text { on } \Gamma,
$$

such that

$$
\begin{aligned}
\|y\|_{L^{2}(\Omega)}^{2} & =(y,-\Delta w)_{\Omega}=(f, w)_{\Omega}-\left(u, \partial_{n} w\right)_{\Gamma} \\
& \leq c\left(\|u\|_{\prod_{j=1}^{N} H_{00}^{1 / 2}\left(\Gamma_{j}\right)^{\prime}}+\|f\|_{\left(H^{2}(\Omega) \cap H_{0}^{1}(\Omega)\right)^{\prime}}\right)\|w\|_{H^{2}(\Omega)},
\end{aligned}
$$

where one uses that the mapping $H^{2}(\Omega) \cap H_{0}^{1}(\Omega) \rightarrow \prod_{j=1}^{N} H_{00}^{1 / 2}\left(\Gamma_{j}\right), w \mapsto \partial_{n} w$, is surjective due to $[13$, Thm. 1.5.2.8]. The desired estimated (2.2) is then obtained by using the a priori estimate $\|w\|_{H^{2}(\Omega)} \leq c\|y\|_{L^{2}(\Omega)}$ and by division by $\|y\|_{L^{2}(\Omega)}$.

The method of proof of this lemma will be revisited in Section 2.5, where we start the discussion of the regularization approach. However, there we will work with different function spaces such that non-convex domains are included in the theory as well. The given proof of Lemma 2.1 is even restricted to convex domains since the isomorphism

$$
\Delta w \in L^{2}(\Omega), w_{\mid \Gamma}=0 \quad \Leftrightarrow \quad w \in H^{2}(\Omega) \cap H_{0}^{1}(\Omega)
$$

is used. If the domain is non-convex one loses at least the uniqueness of the solution $y$ of (2.1). For example, take $\Omega=\left\{(r \cos \theta, r \sin \theta) \in \mathbb{R}^{2}: 0<r<1,0<\theta<\omega\right\}$ and $\lambda=\pi / \omega$, then both $y_{1}=r^{\lambda} \sin (\lambda \theta)$ and $y_{2}=r^{-\lambda} \sin (\lambda \theta)$ are harmonic in $\Omega$ and $y_{1}=y_{2}$ on $\Gamma$. Both satisfy (2.1) with $f \equiv 0$ and $g=y_{1}=y_{2}$ on $\Gamma$. Hence, one needs a larger test space in order to rule out $y_{2}$.

\subsection{Method of transposition in general polygonal domains}

In a first instance we replace the test space $H^{2}(\Omega) \cap H_{0}^{1}(\Omega)$ by

$$
V:=H_{0}^{1}(\Omega) \cap H_{\Delta}^{1}(\Omega) \quad \text { with } \quad H_{\Delta}^{1}(\Omega):=\left\{v \in H^{1}(\Omega): \Delta v \in L^{2}(\Omega)\right\} .
$$

Since $|v|_{H^{1}(\Omega)} \leq c\|\Delta v\|_{L^{2}(\Omega)}$, the graph norm in $V$, that is $\|\Delta v\|_{L^{2}(\Omega)}+|v|_{H^{1}(\Omega)}$, is equivalent to $\|\Delta v\|_{L^{2}(\Omega)}$ such that we will use henceforth

$$
\|v\|_{V}=\|\Delta v\|_{L^{2}(\Omega)} .
$$

Furthermore, let us denote by $V_{\Gamma}$ the space of normal derivatives $\partial_{n} v$ of functions $v \in$ $V$. According to [13, Theorem 1.5.3.10] this space is well-defined and a subspace of $\prod_{j=1}^{N} H_{00}^{1 / 2}\left(\Gamma_{j}\right)^{\prime}$. The natural norm in $V_{\Gamma}$ is given by

$$
\|g\|_{V_{\Gamma}}:=\inf \left\{\|v\|_{V}: v \in V, \partial_{n} v=g\right\} .
$$


In particular, we have for $v \in V$ that

$$
\left\|\partial_{n} v\right\|_{V_{\Gamma}} \leq\|v\|_{V}
$$

Since the previous definitions of the spaces $V$ and $V_{\Gamma}$ are rather formal let us discuss the structure of these spaces.

Remark 2.2. The spaces $V$ and $V_{\Gamma}$ can be characterized as follows:

1. If $\Omega$ is convex the spaces $V$ and $H^{2}(\Omega) \cap H_{0}^{1}(\Omega)$ coincide. However, in non-convex domains the situation is different. In this case there is the splitting

$$
V=\left(H^{2}(\Omega) \cap H_{0}^{1}(\Omega)\right) \oplus \operatorname{Span}\left\{\xi(r) r^{\lambda} \sin (\lambda \theta)\right\},
$$

where $\xi$ denotes a smooth cut-off function which is equal to one in the neighborhood of the non-convex corner. For more details we refer to [14, Sections 1.5, 2.3 and 2.4] and [13, Theorem 4.4.3.7].

2. The mapping $H^{2}(\Omega) \cap H_{0}^{1}(\Omega) \rightarrow \prod_{j=1}^{N} H_{00}^{1 / 2}\left(\Gamma_{j}\right), w \mapsto \partial_{n} w$, is surjective due to [13, Thm. 1.5.2.8]. Accordingly, in the convex case $V_{\Gamma}$ is just $\prod_{j=1}^{N} H_{00}^{1 / 2}\left(\Gamma_{j}\right)$, whereas in the non-convex case there holds

$$
V_{\Gamma}=\left(\prod_{j=1}^{N} H_{00}^{1 / 2}\left(\Gamma_{j}\right)\right) \oplus \operatorname{Span}\left\{\xi(r) r^{\lambda-1}\right\} .
$$

3. In the non-convex case there is $V \hookrightarrow H^{s}(\Omega) \cap H_{0}^{1}(\Omega)$ for $s<1+\lambda$ and $V_{\Gamma} \hookrightarrow H^{t}(\Gamma)$ for $t<\lambda-\frac{1}{2}$. This implies $\left(H^{s}(\Omega) \cap H_{0}^{1}(\Omega)\right)^{\prime} \hookrightarrow V^{\prime}$ and $H^{t}(\Gamma)^{\prime} \hookrightarrow V_{\Gamma}^{\prime}$.

Lemma 2.3. Let $f \in V^{\prime}$ and $u \in V_{\Gamma}^{\prime}$. Then there exists a unique solution

$$
y \in L^{2}(\Omega): \quad(y, \Delta v)_{\Omega}=\left(u, \partial_{n} v\right)_{\Gamma}-(f, v)_{\Omega} \quad \forall v \in V
$$

with

$$
\|y\|_{L^{2}(\Omega)} \leq\|u\|_{V_{\Gamma}^{\prime}}+\|f\|_{V^{\prime}}
$$

Proof. The proof of this lemma is based on the Babuška-Lax-Milgram theorem. Due to (2.5) the right hand side of (2.6) defines a linear functional on $V$. Moreover, the bilinear form is bounded on $L^{2}(\Omega) \times V$. The inf-sup conditions are proved by using the isomorphism

$$
\Delta v \in L^{2}(\Omega), v_{\mid \Gamma}=0 \quad \Leftrightarrow \quad v \in V .
$$

In particular, we obtain by taking $y=\Delta v$

$$
\sup _{y \in L^{2}(\Omega)} \frac{\left|(y, \Delta v)_{\Omega}\right|}{\|y\|_{L^{2}(\Omega)}} \geq \frac{(\Delta v, \Delta v)_{\Omega}}{\|\Delta v\|_{L^{2}(\Omega)}}=\|\Delta v\|_{L^{2}(\Omega)}=\|v\|_{V},
$$


and by taking the solution $v \in V$ of $\Delta v=y$

$$
\sup _{v \in V} \frac{\left|(y, \Delta v)_{\Omega}\right|}{\|v\|_{V}} \geq \frac{(y, y)_{\Omega}}{\|y\|_{L^{2}(\Omega)}}=\|y\|_{L^{2}(\Omega)} .
$$

The existence of the unique solution $y \in L^{2}(\Omega)$ of problem (2.6) follows from the standard Babuška-Lax-Milgram theorem, see for example [3, Theorem 2.1]. The a priori estimate follows from (2.7), and (2.6) and (2.5),

$$
\|y\|_{L^{2}(\Omega)} \leq \sup _{v \in V} \frac{\left|(y, \Delta v)_{\Omega}\right|}{\|v\|_{V}}=\sup _{v \in V} \frac{\left|\left(u, \partial_{n} v\right)_{\Gamma}-(f, v)_{\Omega}\right|}{\|v\|_{V}} \leq\|u\|_{V_{\Gamma}^{\prime}}+\|f\|_{V^{\prime}} .
$$

Note that if a weak solution $y \in\left\{v \in H^{1}(\Omega):\left.v\right|_{\Gamma}=u\right\}$ exists, then with the help of the Green formula

$$
\left(\partial_{n} v, \chi\right)_{\Gamma}=(\nabla v, \nabla \chi)_{\Omega}+(\Delta v, \chi)_{\Omega} \quad \forall v \in H_{\Delta}^{1}(\Omega) \supset V, \forall \chi \in H^{1}(\Omega),
$$

see Lemma 3.4 in the paper [9] by Costabel, it is also a very weak solution. (Set $\chi=y$ and use $\left.(\nabla v, \nabla y)_{\Omega}=(f, v)_{\Omega}.\right)$

A possible difficulty with this formulation is that a conforming discretization with a finite-dimensional space $V_{h} \subset V$ would require the use of $C^{1}$-functions. This is simple for one-dimensional domains $\Omega$ but requires a lot of degrees of freedom in two (and more) dimensions.

We finish this subsection with a regularity result.

Lemma 2.4. The unique solution $y \in L^{2}(\Omega)$ of problem (2.6) with $u \in L^{2}(\Gamma)$ and $f=0$ belongs to $H^{1 / 2}(\Omega)$ and to

$$
\widetilde{W}^{1,2}(\Omega):=\left\{z \in L^{2}(\Omega): \delta^{1 / 2} \nabla z \in L^{2}(\Omega)^{2}\right\}
$$

where $\delta(x)$ is the distance of $x$ to the boundary $\Gamma$. Furthermore, there exists a positive constant $c$ such that

$$
\|y\|_{H^{1 / 2}(\Omega)}+\left\|\delta^{1 / 2} \nabla y\right\|_{L^{2}(\Omega)^{2}} \leq c\|u\|_{L^{2}(\Gamma)} .
$$

Remark 2.5. The book by Chabrowski, [7], deals exclusively with the Dirichlet problem with $L^{2}$ boundary data for elliptic linear equations. The solution is searched there in the Sobolev space (2.9) but domains of class $C^{1,1}$ were considered only.

Proof of Lemma 2.4. In a first step, we use an integral representation and some properties of the layer potentials to get a solution with the appropriate regularity. Theorem 4.2 of Verchota's paper [24] shows that the operator $\frac{1}{2} I+K, K$ being the boundary double layer potential, is an isomorphism from $L^{2}(\Gamma)$ into itself (see also Corollary 4.5 of [19]). According to the trace property for the double layer potential $\mathcal{K}$ (see Section 1 and Corollary 3.2 of [24]), there exists a unique harmonic function $z$ such that

$$
z \rightarrow u \text { a.e. in nontangential cones }
$$


with the notation from [24], and is given by

$$
z=\mathcal{K}\left(\frac{1}{2} I+K\right)^{-1} u .
$$

But due to the above mentioned isomorphism property and Theorem 1 of Costabel's paper [9] (and its following Remark), we obtain that

$$
\|z\|_{H^{1 / 2}(\Omega)} \leq c\|u\|_{L^{2}(\Gamma)} .
$$

Note that Theorems 5.3, 5.4 and Corollary 5.5 of the paper [15] by Jerison and Kenig also yield

$$
\left\|\delta^{1 / 2} \nabla z\right\|_{L^{2}(\Omega)^{2}} \leq c\|u\|_{L^{2}(\Gamma)} .
$$

The second step is to show that $z$ is the very weak solution, hence by uniqueness, we will get $y=z$. The regularity of $y$ and the estimate (2.10) follow from our first step. For that last purpose, we use a density argument. Indeed let $u_{n} \in H^{1}(\Gamma)$ be a sequence of functions such that

$$
u_{n} \rightarrow u \text { in } L^{2}(\Gamma) \text {, as } n \rightarrow \infty .
$$

Consider $z_{n}=\mathcal{K}\left(\frac{1}{2} I+K\right)^{-1} u_{n}$ and let $y_{n} \in L^{2}(\Omega)$ be the unique solution of (2.6) with boundary datum $u_{n}$ and right hand side $f=0$ (that is in $H^{1}(\Omega)$ ). Then by the estimate (2.12) and Lemma 2.3, we get

$$
\begin{gathered}
y_{n} \rightarrow y \text { in } L^{2}(\Omega), \text { as } n \rightarrow \infty, \\
z_{n} \rightarrow z \text { in } H^{1 / 2}(\Omega), \text { as } n \rightarrow \infty .
\end{gathered}
$$

Furthermore by Theorem 5.15 of [15] $z_{n}$ satisfies

$$
\gamma z_{n}=u_{n} \text { on } \Gamma
$$

where $\gamma$ is the trace operator from $H^{1}(\Omega)$ into $H^{1 / 2}(\Gamma)$. Hence we directly deduce that $y_{n}=z_{n}$ and by the above convergence property we conclude that $y=z$.

Corollary 2.6. The unique solution $y \in L^{2}(\Omega)$ of problem (2.6) with $u \in L^{2}(\Gamma)$ and $f \in H^{-1}(\Omega)$ belongs to $H^{1 / 2}(\Omega)$ and to $\widetilde{W}^{1,2}(\Omega)$ from $(2.9)$. There exists a positive constant $c$ such that

$$
\|y\|_{H^{1 / 2}(\Omega)}+\left\|\delta^{1 / 2} \nabla y\right\|_{L^{2}(\Omega)^{2}} \leq c\left(\|u\|_{L^{2}(\Gamma)}+\|f\|_{H^{-1}(\Omega)}\right) .
$$

\subsection{Method of transposition employing weighted Sobolev spaces}

Alternatively to the space $H_{\Delta}^{1}(\Omega) \cap H_{0}^{1}(\Omega)$, one can use the test space

$$
V_{\beta}^{2,2}(\Omega) \cap H_{0}^{1}(\Omega), \quad \beta>1-\frac{\pi}{\omega},
$$


in the non-convex case, where $V_{\beta}^{2,2}(\Omega)$ is a weighted Sobolev space of the class

$$
\begin{aligned}
V_{\beta}^{k, p}(\Omega) & :=\left\{v \in \mathcal{D}^{\prime}(\Omega):\|v\|_{V_{\beta}^{k, p}(\Omega)}<\infty\right\}, \\
\|v\|_{V_{\beta}^{k, p}(\Omega)}^{p} & :=\sum_{|\alpha| \leq k} \int_{\Omega}\left|r^{\beta-k+|\alpha|} D^{\alpha} v\right|^{p},
\end{aligned}
$$

where we use standard multi-index notation. For later use we also introduce

$$
L_{\beta}^{2}(\Omega):=V_{\beta}^{0,2}(\Omega) .
$$

First derivatives of $V_{\beta}^{2,2}(\Omega)$-functions belong to $V_{\beta}^{1,2}(\Omega)$ by definition. The trace space of $V_{\beta}^{1,2}(\Omega)$ is $\prod_{j=1}^{N} V_{\beta}^{1 / 2,2}\left(\Gamma_{j}\right)$, see [18, Lemma 1.2] or [20, Theorem 1.31]. In the next lemma, we will use the spaces

$$
V_{\beta}:=\left\{\begin{array}{ll}
V_{\beta}^{2,2}(\Omega) \cap H_{0}^{1}(\Omega) & \text { for } \omega>\pi, \\
H^{2}(\Omega) \cap H_{0}^{1}(\Omega) & \text { for } \omega<\pi,
\end{array} \quad Y_{\beta}:= \begin{cases}L_{-\beta}^{2}(\Omega) & \text { for } \omega>\pi, \\
L^{2}(\Omega) & \text { for } \omega<\pi,\end{cases}\right.
$$

for $\beta \in\left(1-\frac{\pi}{\omega}, 1\right]$. We endow $V_{\beta}$ with the $V_{\beta}^{2,2}(\Omega)$-norm for $\omega>\pi$ and the $H^{2}(\Omega)$-norm for $\omega<\pi$, as well as $Y_{\beta}$ with the $L_{-\beta}^{2}(\Omega)$-norm for $\omega>\pi$ and the $L^{2}(\Omega)$-norm for $\omega<\pi$.

Remark 2.7. Let us discuss the definition of the space $V_{\beta}$ and the restriction of the weight $\beta$ to the interval $\left(1-\frac{\pi}{\omega}, 1\right]$ in the non-convex case:

1. We require $\beta \in\left(1-\frac{\pi}{\omega}, 1\right]$ in order to have the isomorphism

$$
\Delta v \in L_{\beta}^{2}(\Omega), v_{\mid \Gamma}=0 \quad \Leftrightarrow \quad v \in V_{\beta}
$$

2. It is possible to use the test space $V_{\beta}^{2,2}(\Omega) \cap H_{0}^{1}(\Omega)$ in convex domains as well. However, this implies a loss of information about the solution since this test space is smaller than $H^{2}(\Omega) \cap H_{0}^{1}(\Omega)$ due to the fact that the weight $\beta$ can be negative.

3. Note that $V_{\beta}^{1 / 2,2}\left(\Gamma_{j}\right) \hookrightarrow L^{2}\left(\Gamma_{j}\right)$ for $\beta \leq \frac{1}{2}$. This implies $L^{2}(\Gamma)=\left(\prod_{j=1}^{N} L^{2}\left(\Gamma_{j}\right)\right)^{\prime} \hookrightarrow$ $\left(\prod_{j=1}^{N} V_{\beta}^{1 / 2,2}\left(\Gamma_{j}\right)\right)^{\prime}$. This means that $L^{2}$-boundary data are included in the following discussion if $\beta \leq \frac{1}{2}$.

Lemma 2.8. Let $\beta \in\left(1-\frac{\pi}{\omega}, 1\right]$ and assume that $f \in V_{\beta}^{\prime}$ and $u \in\left(\prod_{j=1}^{N} V_{\beta}^{1 / 2,2}\left(\Gamma_{j}\right)\right)^{\prime}$. Then there exists a unique solution

$$
y \in Y_{\beta} \hookrightarrow L^{2}(\Omega): \quad(y, \Delta v)_{\Omega}=\left(u, \partial_{n} v\right)_{\Gamma}-(f, v)_{\Omega} \quad \forall v \in V_{\beta}
$$

with

$$
\|y\|_{Y_{\beta}} \leq c\left(\|f\|_{V_{\beta}^{\prime}}+\|u\|_{\left(\prod_{j=1}^{N} V_{\beta}^{1 / 2,2}\left(\Gamma_{j}\right)\right)^{\prime}}\right) .
$$


Proof. The convex case was already treated in Lemma 2.1, hence we focus on the nonconvex case. We proceed as in Lemma 2.3. The right hand side of (2.17) defines a continuous functional on $V_{\beta}$. Furthermore, the bilinear form is bounded on $Y_{\beta} \times V_{\beta}$,

$$
\left|(y, \Delta v)_{\Omega}\right| \leq\left\|r^{-\beta} y\right\|_{L^{2}(\Omega)}\left\|r^{\beta} \Delta v\right\|_{L^{2}(\Omega)} \leq\|y\|_{Y_{\beta}}\|v\|_{V_{\beta}} .
$$

The inf-sup conditions for the bilinear form are proved by using the isomorphism (2.16), in particular $\|v\|_{V_{\beta}} \leq c\|\Delta v\|_{L_{\beta}^{2}(\Omega)}$; we obtain by taking $y=r^{2 \beta} \Delta v$

$$
\sup _{y \in Y_{\beta}} \frac{\left|(y, \Delta v)_{\Omega}\right|}{\|y\|_{Y_{\beta}}} \geq \frac{\left(r^{\beta} \Delta v, r^{\beta} \Delta v\right)_{\Omega}}{\left\|r^{2 \beta} \Delta v\right\|_{L_{-\beta}^{2}(\Omega)}}=\frac{\|\Delta v\|_{L_{\beta}^{2}(\Omega)}^{2}}{\|\Delta v\|_{L_{\beta}^{2}(\Omega)}^{2}} \geq c^{-1}\|v\|_{V_{\beta}},
$$

and by taking the solution $v \in V_{\beta}$ of $\Delta v=r^{-2 \beta} y$ with $\|v\|_{V_{\beta}} \leq c\|\Delta v\|_{L_{\beta}^{2}(\Omega)}=c\|y\|_{L_{-\beta}^{2}(\Omega)}$

$$
\sup _{v \in V_{\beta}} \frac{\left|(y, \Delta v)_{\Omega}\right|}{\|v\|_{V_{\beta}}} \geq \frac{\left(r^{-\beta} y, r^{-\beta} y\right)_{\Omega}}{c\|y\|_{L_{-\beta}^{2}(\Omega)}}=c^{-1}\|y\|_{L_{-\beta}^{2}(\Omega)} .
$$

The existence of the unique solution $y \in L_{-\beta}^{2}(\Omega)$ of problem (2.17) follows now from the standard Babuška-Lax-Milgram theorem, see for example [3, Theorem 2.1]. The a priori estimate is obtained with (2.18) and (2.17),

$$
\|y\|_{Y_{\beta}} \leq c \sup _{v \in V_{\beta}} \frac{\left|(y, \Delta v)_{\Omega}\right|}{\|v\|_{V_{\beta}}}=c \sup _{v \in V_{\beta}} \frac{\left|\left(u, \partial_{n} v\right)_{\Gamma}-(f, v)_{\Omega}\right|}{\|v\|_{V_{\beta}}} .
$$

This ends the proof since we already noticed that the enumerator defines a continuous functional on $V_{\beta}$.

\subsection{Berggren's approach}

Berggren's approach [4] avoids test functions in $H_{\Delta}^{1}(\Omega) \cap H_{0}^{1}(\Omega)$ in an explicit way. It can be explained as if we substitute $\varphi:=-\Delta v$ and $\zeta:=\partial_{n} v$ in (2.6),

$$
y \in L^{2}(\Omega): \quad(y, \varphi)_{\Omega}=-(u, \zeta)_{\Gamma}+(f, v)_{\Omega} \quad \forall \varphi \in L^{2}(\Omega) .
$$

The relationship between $\varphi \in L^{2}(\Omega)$ and both $v \in V$ and $\zeta \in V_{\Gamma}$ can be expressed by the weak formulation of the Poisson equation,

$$
v \in V: \quad(\nabla v, \nabla \psi)_{\Omega}=(\varphi, \psi)_{\Omega} \quad \forall \psi \in H_{0}^{1}(\Omega)
$$

and a reformulation of the Green formula (2.8) in the form

$$
\zeta \in V_{\Gamma}: \quad(\zeta, \chi)_{\Gamma}=(\nabla v, \nabla \chi)_{\Omega}-(\varphi, \chi)_{\Omega} \quad \forall \chi \in H^{1}(\Omega) \backslash H_{0}^{1}(\Omega) .
$$

Note that Berggren's formulation is not a system with three unknown functions since the second and third equations compute actions on the test function $\varphi$. Indeed, let $S: L^{2}(\Omega) \rightarrow V$ and $F: L^{2}(\Omega) \rightarrow V_{\Gamma}$ be the solution operators of (2.20) and (2.21), respectively, defined by $S \varphi:=v$ and $F \varphi:=\zeta$, then we could also write

$$
y \in L^{2}(\Omega): \quad(y, \varphi)_{\Omega}=-(u, F \varphi)_{\Gamma}-(f, S \varphi)_{\Omega} \quad \forall \varphi \in L^{2}(\Omega)
$$

instead of (2.19). 
Lemma 2.9. Berggren's formulation (2.19), (2.20), (2.21) is equivalent to the formulation (2.6).

Proof. We first assume that $y \in L^{2}(\Omega)$ satisfies (2.6) and show (2.19)-(2.21). For any $\varphi \in L^{2}(\Omega)$ let $v$ be the variational solution of $-\Delta v=\varphi$ defined by (2.20), hence $v \in V$ and $\zeta:=\partial_{n} v \in V_{\Gamma}$. Based on the formula (2.8), we obtain (2.21). With (2.6) we finally get also (2.19).

Let now $y$ satisfy (2.19)-(2.21). Since $\varphi \in L^{2}(\Omega)$ we get from (2.20) that $v \in V$. Moreover, we obtain $\zeta=\partial_{n} v \in V_{\Gamma}$ from (2.21). Hence equation (2.19) becomes

$$
-(y, \Delta v)_{\Omega}=-\left(u, \partial_{n} v\right)_{\Gamma}+(f, v)_{\Omega} \quad \forall v \in V
$$

due to the isometry between $L^{2}(\Omega)$ and $V$.

Remark 2.10. Berggren used the regularity $v \in H^{3 / 2+\epsilon}(\Omega)$ with some $\epsilon>0$ which implies $\zeta \in H^{\epsilon}(\Gamma)$. However, he did not consider the maximal domain of the elliptic operator, i.e., $v \in V$ and $\zeta \in V_{\Gamma}$. But with the explanations of Subsection 2.2 these regularities should be obvious. Thus the result of Lemma 2.9 is slightly more general than that of Berggren.

\subsection{The regularization approach}

A further idea is to regularize the boundary data and then to apply standard methods. This approach has already been considered within the proof of Lemma 2.1. In contrast, we do not use the isomorphism

$$
\Delta w \in L^{2}(\Omega), w_{\mid \Gamma}=0 \quad \Leftrightarrow \quad w \in H^{2}(\Omega) \cap H_{0}^{1}(\Omega),
$$

which can only be employed in case of convex domains, but the isomorphism

$$
\Delta v \in L^{2}(\Omega), v_{\mid \Gamma}=0 \quad \Leftrightarrow \quad v \in V .
$$

This allows us to apply the regularization approach in the non-convex case as well. Moreover, we propose two different strategies how the regularized Dirichlet boundary data can be constructed in an explicit way. Thereby we will be able in Subsection 3.2 to calculate approximate solutions of the regularized problems based on a finite element method. For the data we assume henceforth $u \in L^{2}(\Gamma)$ and $f \in H^{-1}(\Omega)$. This is not only for simplicity but also due to the fact that already for Dirichlet boundary data in $L^{2}(\Gamma)$ the convergence rates of the approximate solutions in Subsection 3.2 tend to zero as the maximal interior angle tends to $2 \pi$.

We start with general convergence results for the regularized solutions. To this end let $u^{h} \in H^{1 / 2}(\Gamma)$ be a sequence of functions such that

$$
\lim _{h \rightarrow 0}\left\|u-u^{h}\right\|_{L^{2}(\Gamma)}=0 .
$$

Let now $y^{h} \in Y_{*}^{h}:=\left\{v \in H^{1}(\Omega):\left.v\right|_{\Gamma}=u^{h}\right\}$ be the variational solution,

$$
y^{h} \in Y_{*}^{h}: \quad\left(\nabla y^{h}, \nabla v\right)_{\Omega}=(f, v)_{\Omega} \quad \forall v \in H_{0}^{1}(\Omega) .
$$


Lemma 2.11. Let $u \in L^{2}(\Gamma)$ and $f \in H^{-1}(\Omega)$. Then the limit $y:=\lim _{h \rightarrow 0} y^{h}$ exists, belongs to $L^{2}(\Omega)$, and is the very weak solution, that means it satisfies (2.6).

Proof. First we show that $y^{h}$ is a Cauchy sequence in $L^{2}(\Omega)$. From (2.22) and Green's formula, we have for any $v \in V$,

$$
\begin{gathered}
(f, v)_{\Omega}=\left(\nabla y^{h}, \nabla v\right)_{\Omega}=-\left(y^{h}, \Delta v\right)_{\Omega}+\left(y^{h}, \partial_{n} v\right)_{\Gamma}, \\
(f, v)_{\Omega}=\left(\nabla y^{h^{\prime}}, \nabla v\right)_{\Omega}=-\left(y^{h^{\prime}}, \Delta v\right)_{\Omega}+\left(y^{h^{\prime}}, \partial_{n} v\right)_{\Gamma} .
\end{gathered}
$$

Hence due to $y^{h}=u^{h}$ and $y^{h^{\prime}}=u^{h^{\prime}}$ on $\Gamma$, we deduce that

$$
\left(y^{h}-y^{h^{\prime}}, \Delta v\right)_{\Omega}=\left(u^{h}-u^{h^{\prime}}, \partial_{n} v\right)_{\Gamma} \quad \forall v \in V .
$$

Now for any $z \in L^{2}(\Omega)$, let $v_{z} \in V$ be such that

$$
\Delta v_{z}=z
$$

that clearly satisfies

$$
\left\|\partial_{n} v_{z}\right\|_{L^{2}(\Gamma)} \leq c\left\|v_{z}\right\|_{H^{s}(\Omega)} \leq c\|z\|_{L^{2}(\Omega)}
$$

with some $s \in\left(\frac{3}{2}, 1+\lambda\right), s \leq 2$. Finally, we obtain with (2.23) and (2.25)

$$
\begin{aligned}
\left\|y^{h}-y^{h^{\prime}}\right\|_{L^{2}(\Omega)} & =\sup _{z \in L^{2}(\Omega), z \neq 0} \frac{\left(y^{h}-y^{h^{\prime}}, z\right)_{\Omega}}{\|z\|_{L^{2}(\Omega)}}=\sup _{z \in L^{2}(\Omega), z \neq 0} \frac{\left(u^{h}-u^{h^{\prime}}, \partial_{n} v_{z}\right)_{\Gamma}}{\|z\|_{L^{2}(\Omega)}} \\
& \leq\left\|u^{h}-u^{h^{\prime}}\right\|_{L^{2}(\Gamma)} \sup _{z \in L^{2}(\Omega), z \neq 0} \frac{\left\|\partial_{n} v_{z}\right\|_{L^{2}(\Gamma)}}{\|z\|_{L^{2}(\Omega)}}=c\left\|u^{h}-u^{h^{\prime}}\right\|_{L^{2}(\Gamma)} .
\end{aligned}
$$

Since $u^{h}$ converges in $L^{2}(\Gamma)$, it is a Cauchy sequence and hence also $y^{h}$ is a Cauchy sequence and converges in $L^{2}(\Omega)$ by the completeness of $L^{2}(\Omega)$.

From $V \subset H_{0}^{1}(\Omega)$ we obtain by $(2.22)$ and the Green formula (2.8)

$$
\begin{aligned}
(f, v)_{\Omega} & =\left(\nabla y^{h}, \nabla v\right)_{\Omega}=\left(\Delta v, y^{h}\right)_{\Omega}-\left(\partial_{n} v, y^{h}\right)_{\Gamma} \\
& =\left(\Delta v, y^{h}\right)_{\Omega}-\left(\partial_{n} v, u^{h}\right)_{\Gamma} \quad \forall v \in V .
\end{aligned}
$$

Since $\Delta v \in L^{2}(\Omega)$ and $\partial_{n} v \in L^{2}(\Gamma)$ we can pass to the limit and obtain that the limit function $y$ satisfies (2.6).

We can estimate the regularization error by a similar technique.

Lemma 2.12. Let $s=\frac{1}{2}$ if $\Omega$ is convex and $s \in\left[0, \lambda-\frac{1}{2}\right)$ if $\Omega$ is non-convex. Then the estimate

$$
\left\|y-y^{h}\right\|_{L^{2}(\Omega)} \leq c\left\|u-u^{h}\right\|_{H^{-s}(\Gamma)}
$$

holds. 
Proof. We use the approach of the proof of Lemma 2.11 and just replace $u^{h^{\prime}}$ by $u$ and $y^{h^{\prime}}$ by $y$ to get

$$
\left(y-y^{h}, \Delta v\right)_{\Omega}=\left(u-u^{h}, \partial_{n} v\right)_{\Gamma} \quad \forall v \in V .
$$

Again, for any $z \in L^{2}(\Omega)$, we let $v_{z} \in V$ be such that $\Delta v_{z}=z$ but estimate now in a sharper way

$$
\left\|\partial_{n} v_{z}\right\|_{H^{s}(\Gamma)} \leq c\left\|v_{z}\right\|_{H^{s+3 / 2}(\Omega)} \leq c\|z\|_{L^{2}(\Omega)}
$$

As in the previous proof we get

$$
\begin{aligned}
\left\|y-y^{h}\right\|_{L^{2}(\Omega)} & =\sup _{z \in L^{2}(\Omega), z \neq 0} \frac{\left(u-u^{h}, \partial_{n} v_{z}\right)_{\Omega}}{\|z\|_{L^{2}(\Omega)}} \\
& \leq\left\|u-u^{h}\right\|_{H^{-s}(\Gamma)} \sup _{z \in L^{2}(\Omega), z \neq 0} \frac{\left\|\partial_{n} v_{z}\right\|_{H^{s}(\Gamma)}}{\|z\|_{L^{2}(\Omega)}} \\
& \leq c\left\|u-u^{h}\right\|_{H^{-s}(\Gamma)} .
\end{aligned}
$$

Actually, the proof is for $s>0$ but of course the statement holds when $s$ is decreased.

A choice for the construction of the regularized function $u^{h}$ could be the use of the $L^{2}(\Gamma)$-projection $\Pi_{h} u$ into a piecewise polynomial space on the boundary (which we call $Y_{h}^{\partial}$ in Section 3) or the use of the Carstensen interpolant $C_{h} u$, see [5]. Namely, if $\mathcal{N}_{\Gamma}$ is the set of nodes of the triangulation on the boundary, we set

$$
C_{h} u=\sum_{x \in \mathcal{N}_{\Gamma}} \pi_{x}(u) \lambda_{x}
$$

where $\lambda_{x}$ is the standard hat function related to $x$ and

$$
\pi_{x}(u)=\frac{\int_{\Gamma} u \lambda_{x}}{\int_{\Gamma} \lambda_{x}}=\frac{\left(u, \lambda_{x}\right)_{\Gamma}}{\left(1, \lambda_{x}\right)_{\Gamma}}
$$

The advantages of the interpolant in comparison with the $L^{2}$-projection are its local definition and the property

$$
u \in[a, b] \quad \Rightarrow \quad C_{h} u \in[a, b],
$$

see [10]; a disadvantage for our application in optimal control is that $C_{h} u_{h} \neq u_{h}$ for piecewise linear $u_{h}$. We prove now regularization error estimates for the case that the regularized function $u^{h}$ is constructed via $C_{h} u$ or $\Pi_{h} u$.

Lemma 2.13. If $u^{h}$ is the piecewise linear Carstensen interpolant of $u$ or the $L^{2}(\Gamma)$ projection of $u$ into a space of piecewise linear functions, then there holds

$$
\left\|u-u^{h}\right\|_{H^{-s}(\Gamma)} \leq c h^{s}\|u\|_{L^{2}(\Gamma)}, \quad s \in[0,1]
$$

as well as

$$
\left\|u^{h}\right\|_{L^{2}(\Gamma)} \leq c\|u\|_{L^{2}(\Gamma)} .
$$


Proof. The interpolation error estimate and stability result are derived in [5, 10] for domains. The proofs can be transferred to estimates on the boundary $\Gamma$. For the sake of completeness we sketch these proofs in the Appendix, see Lemma A.2.

In the case of the $L^{2}$-projection, the second estimate holds with constant one. For the first estimate, we notice that by using the properties of the $L^{2}$-projection, we have

$$
\begin{aligned}
\left(u-\Pi_{h} u, \varphi\right)_{\Gamma} & =\left(u-\Pi_{h} u, \varphi-\Pi_{h} \varphi\right)_{\Gamma}=\left(u, \varphi-\Pi_{h} \varphi\right)_{\Gamma} \leq\|u\|_{L^{2}(\Gamma)}\left\|\varphi-\Pi_{h} \varphi\right\|_{L^{2}(\Gamma)} \\
& \leq\|u\|_{L^{2}(\Gamma)}\left\|\varphi-C_{h} \varphi\right\|_{L^{2}(\Gamma)} \leq c h^{s}\|u\|_{L^{2}(\Gamma)}\|\varphi\|_{H^{s}(\Gamma)}
\end{aligned}
$$

where we used Lemma A.2 in the last step. We conclude

$$
\left\|u-u^{h}\right\|_{H^{-s}(\Gamma)}=\sup _{\varphi \in H^{s}(\Gamma), \varphi \neq 0} \frac{\left(u-\Pi_{h} u, \varphi\right)_{\Gamma}}{\|\varphi\|_{H^{s}(\Gamma)}} \leq c h^{s}\|u\|_{L^{2}(\Gamma)} .
$$

which is the assertion.

By setting $s=0$ in the previous lemma we obtain

$$
\left\|u-u^{h}\right\|_{L^{2}(\Gamma)} \leq c\|u\|_{L^{2}(\Gamma)}
$$

for the different choices of the regularized function $u^{h}$. This means that for $u \in L^{2}(\Gamma)$ the difference $u-u^{h}$ is uniformly bounded in $L^{2}(\Gamma)$ independent of $h$. However, we require strong convergence in $L^{2}(\Gamma)$ for $u \in L^{2}(\Gamma)$, i.e.

$$
\lim _{h \rightarrow 0}\left\|u-u^{h}\right\|_{L^{2}(\Gamma)}=0 .
$$

This is subject of the next lemma. A comparable result for the Ritz-projection can be found in e.g. [8, Theorem 3.2.3].

Lemma 2.14. Let $u \in L^{2}(\Gamma)$ and let $u^{h}$ be the piecewise linear Carstensen interpolant of $u$ or the $L^{2}(\Gamma)$-projection of $u$ into a space of piecewise linear functions. Then there holds

$$
\lim _{h \rightarrow 0}\left\|u-u^{h}\right\|_{L^{2}(\Gamma)}=0 .
$$

Proof. We show the validity of this lemma for the Carstensen interpolant. The convergence result for the $L^{2}(\Gamma)$-projection can be proven analogously.

Due to the compact embedding $H^{1}(\Gamma) \stackrel{c}{\hookrightarrow} L^{2}(\Gamma)$ there exists a sequence of functions $u_{n} \in H^{1}(\Gamma)$ such that

$$
\lim _{n \rightarrow \infty}\left\|u-u_{n}\right\|_{L^{2}(\Gamma)}=0
$$

with

$$
\left\|u_{n}\right\|_{H^{1}(\Gamma)} \leq c_{n},
$$

where the constant $c_{n}$ may depend on $n$. Thus, for every $\varepsilon>0$ there is a positive integer $N$ such that

$$
\left\|u-u_{n}\right\|_{L^{2}(\Gamma)} \leq \frac{\varepsilon}{2}
$$


for $n \geq N$. By inserting the function $u_{n}$ and its Carstensen interpolant $C_{h} u_{n}$ as intermediate functions into the desired term we obtain

$$
\left\|u-C_{h} u\right\|_{L^{2}(\Gamma)} \leq\left\|u-u_{n}\right\|_{L^{2}(\Gamma)}+\left\|u_{n}-C_{h} u_{n}\right\|_{L^{2}(\Gamma)}+\left\|C_{h}\left(u_{n}-u\right)\right\|_{L^{2}(\Gamma)} .
$$

The results of Lemma 2.13 and the inequalities (2.28) and (2.29) imply

$$
\left\|u-C_{h} u\right\|_{L^{2}(\Gamma)} \leq c\left(\frac{\varepsilon}{2}+h c_{n}\right) .
$$

Since for every constant $c_{n}$ there is a parameter $h_{n}$ such that $h c_{n} \leq \frac{\varepsilon}{2}$ for all $h \leq h_{n}$ we arrive at

$$
\left\|u-C_{h} u\right\|_{L^{2}(\Gamma)} \leq c \varepsilon
$$

and the desired result follows.

\subsection{A negative result}

Since the boundary datum $u \in L^{2}(\Gamma) \hookrightarrow L^{1}(\Gamma)$ is trace of a function $w \in W^{1,1}(\Omega)$ an idea could be to search $y=y_{0}+w$ with $\left.y_{0}\right|_{\Gamma}=0$ and

$$
y_{0} \in W_{0}^{1,1}(\Omega): \quad\left(\nabla y_{0}, \nabla v\right)_{\Omega}=(f, v)_{\Omega}-(\nabla w, \nabla v)_{\Omega}=:(F, v)_{\Omega} \quad \forall v \in W_{0}^{1, \infty}(\Omega) .
$$

However, bilinear forms $a(u, v): W_{0}^{1, p}(\Omega) \times W_{0}^{1, p^{\prime}}(\Omega) \rightarrow \mathbb{C}$ are investigated in $[22,23]$ and it is shown that

$$
\forall F \in W^{1, p^{\prime}}(\Omega)^{*} \quad \exists z \in W_{0}^{1, p}(\Omega): \quad a(z, v)=(F, v)_{\Omega} \quad \forall v \in W_{0}^{1, p^{\prime}}(\Omega)
$$

holds for $p \in(1, \infty)$ only since $c_{p} \rightarrow \infty$ for $p \rightarrow 1$ in the inf-sup condition

$$
c_{p} \sup _{\phi \in W_{0}^{1, p^{\prime}}(\Omega)} \frac{(\nabla z, \nabla \phi)_{\Omega}}{\|\nabla \phi\|_{L^{p^{\prime}}(\Omega)}} \geq\|\nabla z\|_{L^{p}(\Omega)} \quad \text { for } 1<p<\infty .
$$

\section{Discretization of the boundary value problem}

Let $\left(\mathcal{T}_{h}\right)_{h>0}$ be a family of conforming, quasi-uniform finite element meshes, and introduce the finite element spaces

$$
Y_{h}=\left\{v_{h} \in H^{1}(\Omega):\left.v_{h}\right|_{T} \in \mathcal{P}_{1} \forall T \in \mathcal{T}_{h}\right\}, \quad Y_{0 h}=Y_{h} \cap H_{0}^{1}(\Omega), \quad Y_{h}^{\partial}=\left.Y_{h}\right|_{\partial \Omega} .
$$

\subsection{Berggren's approach}

Let $u \in L^{2}(\Gamma)$ and $f \in H^{-1}(\Omega)$. We discretize the formulation (2.19), (2.20), (2.21) in a straightforward manner,

$$
\begin{array}{rlrlrl}
y_{h} & \in Y_{h}: & \left(y_{h}, \varphi_{h}\right)_{\Omega} & =-\left(u, \zeta_{h}\right)_{\Gamma}+\left(f, v_{h}\right)_{\Omega} & & \forall \varphi_{h} \in Y_{h}, \\
v_{h} \in Y_{0 h}: & \left(\nabla v_{h}, \nabla \psi_{h}\right)_{\Omega}=\left(\varphi_{h}, \psi_{h}\right)_{\Omega} & & \forall \psi_{h} \in Y_{0 h}, \\
\zeta_{h} \in Y_{h}^{\partial}: & \left(\zeta_{h}, \chi_{h}\right)_{\Gamma}=\left(\nabla v_{h}, \nabla \chi_{h}\right)_{\Omega}-\left(\varphi_{h}, \chi_{h}\right)_{\Omega} & & \forall \chi_{h} \in Y_{h} \backslash Y_{0 h} .
\end{array}
$$


Note that $\zeta_{h} \neq F \varphi_{h}$ and $v_{h} \neq S \varphi_{h}$ with $F$ and $S$ from Subsection 2.4, hence it is only an approximate Galerkin formulation.

Berggren showed in Theorem 5.2 of [4] that this formulation is equivalent with the standard finite element approximation with $L^{2}$-projection of the boundary data,

$$
\begin{aligned}
& y_{h} \in Y_{h}: \quad\left(\nabla y_{h}, \nabla \varphi_{h}\right)_{\Omega}=\left(f, \varphi_{h}\right)_{\Omega} \quad \forall \varphi_{h} \in Y_{0 h}, \\
& \left(y_{h}, \varphi_{h}\right)_{\Gamma}=\left(u, \varphi_{h}\right)_{\Gamma} \quad \forall \varphi_{h} \in Y_{h}^{\partial} .
\end{aligned}
$$

Note that $y_{h}=u_{h}$ on $\Gamma$ when $u=u_{h} \in Y_{h}^{\partial}$. This will be of interest in the discretization of optimal control problems.

Berggren proved also the following discretization error estimate, see Theorem 5.5 of [4].

Lemma 3.1. Let $\omega$ be the maximal interior angle of the domain $\Omega$, and denote by $\lambda=\pi / \omega$ the corresponding singularity exponent. Let $s^{\prime} \in\left(0, \frac{1}{2}\right]$ be a real number with $s^{\prime}<\lambda-\frac{1}{2}$, and let $s \in\left[0, s^{\prime}\right)$ be a further real number. Then the error estimate

$$
\left\|y-y_{h}\right\|_{L^{2}(\Omega)} \leq c\left(h^{s}\|y\|_{H^{s}(\Omega)}+h^{s^{\prime}}\|u\|_{L^{2}(\Gamma)}+h^{s^{\prime}+1 / 2}\|f\|_{H^{-1}(\Omega)}\right)=\mathcal{O}\left(h^{s}\right)
$$

holds, this means that we have convergence order $s$,

$$
s=\min \left\{\frac{1}{2}, \lambda-\frac{1}{2}\right\}-\varepsilon= \begin{cases}\frac{1}{2}-\varepsilon \quad \text { for convex domains } \\ \lambda-\frac{1}{2}-\varepsilon & \text { for non-convex domains }\end{cases}
$$

$\varepsilon>0$ arbitrary.

Note that $s \rightarrow 0$ for $\omega \rightarrow 2 \pi$.

We will show in the next section that in the convex case the convergence order is $\frac{1}{2}$, without $\varepsilon$.

\subsection{Regularization approach}

We consider a regularization strategy such that $u^{h} \in Y_{h}^{\partial}$, see Subsection 2.5. Recall that the corresponding solution $y^{h} \in Y_{*}^{h}:=\left\{v \in H^{1}(\Omega):\left.v\right|_{\Gamma}=u^{h}\right\}$ is defined via (2.22). For a regularization using the $L^{2}(\Gamma)$-projection or the Carstensen interpolant we have the regularization error estimate

$$
\left\|y-y^{h}\right\|_{L^{2}(\Omega)} \leq c\left\|u-u^{h}\right\|_{H^{-s}(\Gamma)} \leq c h^{s}\|u\|_{L^{2}(\Gamma)}
$$

with $s=\frac{1}{2}$ if $\Omega$ is convex and $s \in\left[0, \lambda-\frac{1}{2}\right)$ if $\Omega$ is non-convex, see Lemmas 2.12 and 2.13 .

The finite element solution $y_{h}$ is now searched in $Y_{* h}:=Y_{*}^{h} \cap Y_{h}$ and is defined in the classical way,

$$
y_{h} \in Y_{* h}: \quad\left(\nabla y_{h}, \nabla v_{h}\right)_{\Omega}=\left(f, v_{h}\right)_{\Omega} \quad \forall v_{h} \in Y_{0 h}
$$

Note that, if we construct $u^{h}$ by the $L^{2}$-projection, we recover the Berggren approach as a special case. 
Lemma 3.2. The finite element error estimate

$$
\left\|y^{h}-y_{h}\right\|_{L^{2}(\Omega)} \leq \operatorname{ch}^{s}\left(h^{1 / 2}\|f\|_{H^{-1}(\Omega)}+\|u\|_{L^{2}(\Gamma)}\right)
$$

holds for $s=\frac{1}{2}$ in the convex case and for $s<\lambda-\frac{1}{2}$ in the non-convex case.

Before we prove this lemma we can immediately imply the following final error estimate for this approach. By the triangle inequality we have

$$
\left\|y-y_{h}\right\|_{L^{2}(\Omega)} \leq\left\|y-y^{h}\right\|_{L^{2}(\Omega)}+\left\|y^{h}-y_{h}\right\|_{L^{2}(\Omega)} .
$$

The first term is already treated in (3.4). The second term is treated in Lemma 3.2.

Corollary 3.3. The discretization error estimate

$$
\left\|y-y_{h}\right\|_{L^{2}(\Omega)} \leq \operatorname{ch}^{s}\left(h^{1 / 2}\|f\|_{H^{-1}(\Omega)}+\|u\|_{L^{2}(\Gamma)}\right)
$$

holds for $s=\frac{1}{2}$ in the convex case and $s \in\left[0, \lambda-\frac{1}{2}\right)$ in the non-convex case.

Note that the order can be improved if the boundary datum $u$ is more regular, see Remark 5.3.

Proof of Lemma 3.2. Let $B_{h} u^{h} \in Y_{* h}$ be the discrete harmonic extension defined by

$$
\left(\nabla B_{h} u^{h}, \nabla v_{h}\right)_{\Omega}=0 \quad \forall v_{h} \in Y_{0 h}
$$

which satisfies

$$
\left\|\nabla B_{h} u^{h}\right\|_{L^{2}(\Omega)} \leq c\left\|u^{h}\right\|_{H^{1 / 2}(\Gamma)} \leq c h^{-1 / 2}\left\|u^{h}\right\|_{L^{2}(\Gamma)} \leq c h^{-1 / 2}\|u\|_{L^{2}(\Gamma)} .
$$

The first estimate can be cited from [17, Lemma 3.2], the second follows from an inverse inequality, the third from Lemma 2.13.

Now we notice that

$$
y^{h}=B_{h} u^{h}+y_{0}^{h} \quad \text { as well as } \quad y_{h}=B_{h} u^{h}+y_{0 h},
$$

where $y_{0}^{h} \in H_{0}^{1}(\Omega)$ and $y_{0 h} \in Y_{0 h}$ satisfy

$$
\begin{aligned}
\left(\nabla y_{0}^{h}, \nabla v\right)_{\Omega} & =(f, v)_{\Omega}-\left(\nabla\left(B_{h} u^{h}\right), \nabla v\right)_{\Omega} \quad \forall v \in H_{0}^{1}(\Omega), \\
\left(\nabla y_{0 h}, \nabla v_{h}\right)_{\Omega} & =\left(f, v_{h}\right)_{\Omega}-\left(\nabla\left(B_{h} u^{h}\right), \nabla v_{h}\right)_{\Omega} \quad \forall v_{h} \in Y_{0 h} .
\end{aligned}
$$

Hence $y_{0 h}$ is the Galerkin approximation of $y_{0}^{h}$ and therefore (since $Y_{0 h} \subset H_{0}^{1}(\Omega)$ )

$$
\left(\nabla\left(y_{0}^{h}-y_{0 h}\right), \nabla v_{h}\right)_{\Omega}=0 \quad \forall v_{h} \in Y_{0 h} .
$$

By taking $v=y_{0}^{h}$ in (3.7) (resp. $v_{h}=y_{0 h}$ in (3.8)), we see that

$$
\begin{array}{r}
\left\|\nabla y_{0}^{h}\right\|_{L^{2}(\Omega)}^{2} \leq\|f\|_{H^{-1}(\Omega)}\left\|y_{0}^{h}\right\|_{H^{1}(\Omega)}+\left\|\nabla\left(B_{h} u^{h}\right)\right\|_{L^{2}(\Omega)}\left\|\nabla y_{0}^{h}\right\|_{L^{2}(\Omega)}, \\
\left\|\nabla y_{0 h}\right\|_{L^{2}(\Omega)}^{2} \leq\|f\|_{H^{-1}(\Omega)}\left\|y_{0 h}\right\|_{H^{1}(\Omega)}+\left\|\nabla\left(B_{h} u^{h}\right)\right\|_{L^{2}(\Omega)}\left\|\nabla y_{0 h}\right\|_{L^{2}(\Omega)} .
\end{array}
$$


By the Poincaré inequality we obtain

$$
\begin{aligned}
& \left\|\nabla y_{0}^{h}\right\|_{L^{2}(\Omega)} \leq c\left(\|f\|_{H^{-1}(\Omega)}+\left\|\nabla\left(B_{h} u^{h}\right)\right\|_{L^{2}(\Omega)}\right), \\
& \left\|\nabla y_{0 h}\right\|_{L^{2}(\Omega)} \leq c\left(\|f\|_{H^{-1}(\Omega)}+\left\|\nabla\left(B_{h} u^{h}\right)\right\|_{L^{2}(\Omega)}\right) .
\end{aligned}
$$

With the help of (3.6) we arrive at

$$
\left\|\nabla y_{0}^{h}\right\|_{L^{2}(\Omega)}+\left\|\nabla y_{0 h}\right\|_{L^{2}(\Omega)} \leq c\left(\|f\|_{H^{-1}(\Omega)}+h^{-1 / 2}\|u\|_{L^{2}(\Gamma)}\right) .
$$

Now as before we start with

$$
\left\|y_{0}^{h}-y_{0 h}\right\|_{L^{2}(\Omega)}=\sup _{z \in L^{2}(\Omega), z \neq 0} \frac{\left(y_{0}^{h}-y_{0 h}, z\right)_{\Omega}}{\|z\|_{L^{2}(\Omega)}} .
$$

Letting again $v_{z} \in V$ be such that $\Delta v_{z}=z$, we get

$$
\left\|y_{0}^{h}-y_{0 h}\right\|_{L^{2}(\Omega)}=\sup _{z \in L^{2}(\Omega), z \neq 0} \frac{\left(\nabla\left(y_{0}^{h}-y_{0 h}\right), \nabla v_{z}\right)_{\Omega}}{\|z\|_{L^{2}(\Omega)}}
$$

and therefore thanks to (3.9) we arrive at

$$
\left\|y_{0}^{h}-y_{0 h}\right\|_{L^{2}(\Omega)}=\sup _{z \in L^{2}(\Omega), z \neq 0} \frac{\left(\nabla\left(y_{0}^{h}-y_{0 h}\right), \nabla\left(v_{z}-I_{h} v_{z}\right)\right)_{\Omega}}{\|z\|_{L^{2}(\Omega)}},
$$

where $I_{h}$ is the standard Lagrange interpolation operator. By the Cauchy-Schwarz inequality, the well-known estimate

$$
\left\|v_{z}-I_{h} v_{z}\right\|_{1, \Omega} \leq c h^{1 / 2+s}\left\|v_{z}\right\|_{H^{3 / 2+s}(\Omega)} \leq c h^{1 / 2+s}\|z\|_{L^{2}(\Omega)},
$$

with $s=\frac{1}{2}$ in the convex case and $s<\lambda-\frac{1}{2}$ in the non-convex case, and the estimate (2.27) we obtain

$$
\left\|y_{0}^{h}-y_{0 h}\right\|_{L^{2}(\Omega)} \leq c h^{1 / 2+s}\left\|\nabla\left(y_{0}^{h}-y_{0 h}\right)\right\|_{L^{2}(\Omega)} .
$$

Using the a priori estimate (3.10), we conclude that

$$
\left\|y_{0}^{h}-y_{0 h}\right\|_{L^{2}(\Omega)} \leq c h^{s}\left(h^{1 / 2}\|f\|_{H^{-1}(\Omega)}+\|u\|_{L^{2}(\Gamma)}\right) .
$$

Note that we have now proved the convergence order $\frac{1}{2}$ for Berggren's approach, in the convex case. 


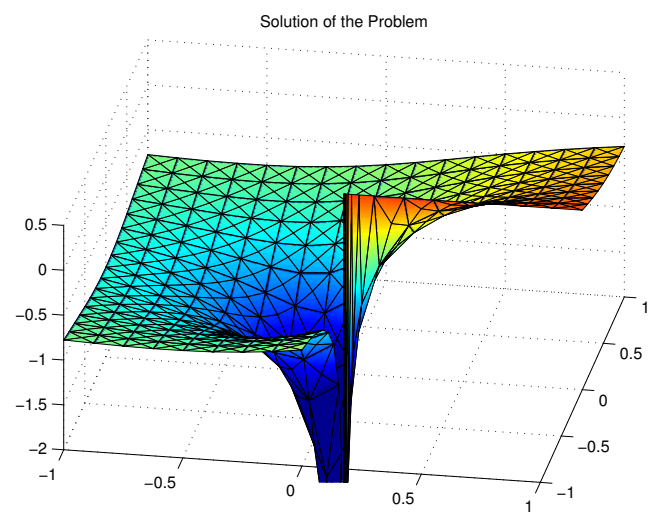

Figure 1: Visualization of the exact solution

\section{Numerical test}

This section is devoted to the numerical verification of our theoretical results. For that purpose we present an example with known solution. Furthermore, to examine the influence of the corner singularities, we consider different polygonal domains $\Omega_{\omega}$ depending on an interior angle $\omega \in(0,2 \pi)$. These domains are defined by

$$
\Omega_{\omega}:=(-1,1)^{2} \cap\left\{x \in \mathbb{R}^{2}:(r(x), \varphi(x)) \in(0, \sqrt{2}] \times[0, \omega]\right\},
$$

where $r$ and $\varphi$ stand for the polar coordinates located at the origin. The boundary of $\Omega_{\omega}$ is denoted by $\Gamma_{\omega}$ which is decomposed into straight line segments $\Gamma_{j}, j=1, \ldots, m(\omega)$, counting counterclockwise beginning at the origin. As numerical example we consider the problem

$$
\begin{aligned}
-\Delta y=0 & \text { in } \Omega_{\omega}, \\
y=u & \text { on } \Gamma_{j}, \quad j=1, \ldots, m(\omega) .
\end{aligned}
$$

The boundary datum $u$ is chosen as

$$
u:=r^{-0.4999} \sin (-0.4999 \varphi) \quad \text { on } \Gamma_{\omega} .
$$

This function belongs to $L^{p}(\Gamma)$ for every $p<2.0004$. The exact solution of our problem is simply

$$
y=r^{-0.4999} \sin (-0.4999 \varphi),
$$

since $y$ is harmonic. A plot of it can be seen in Figure 1. We solve the problem numerically by using a finite element method with piecewise linear finite elements combined with either the $L^{2}$-projection or the Carstensen interpolant of the data on the boundary. The finite element meshes for the calculations are generated by using a newest vertex bisection algorithm as described in [21]. The discretization errors for different mesh sizes and the experimental orders of convergence are given in Tables 1-3 below for interior angles $\omega \in\{3 \pi / 4,3 \pi / 2,355 \pi / 180\}$, where the discrete solutions based on the $L^{2}$-projection 


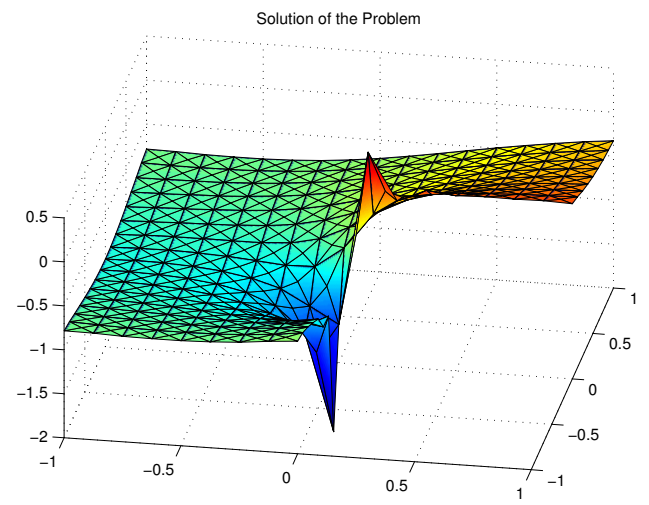

(a) Using $L^{2}$-projection: $u^{h}=\Pi_{h} u$

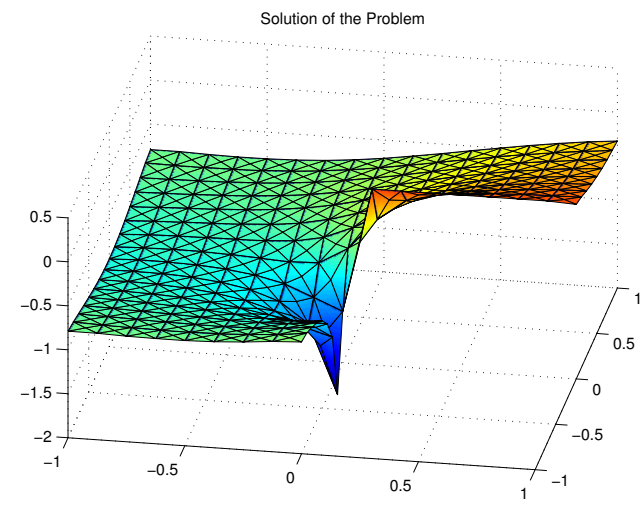

(b) Using Carstensen interpolant: $u^{h}=C_{h} u$

Figure 2: Visualization of the approximate solutions with $h=1 / 8$

and the Carstensen interpolant are denoted by $y_{h, 2}$ or $y_{h, C}$, respectively. We note that the errors are calculated by an adaptive quadrature formula. Apparently, the results are very much in congruence with the predicted orders. The different numerical solutions $y_{h, 2}$ and $y_{h, C}$ for $\omega=3 \pi / 2$ and $h=1 / 8$ are displayed in Figure 2a and Figure 2b, respectively. We see that the infinite boundary value in the origin is replaced by a finite one. If using the $L^{2}$-projection of the data, we see also that the zero boundary values at the edge with $\theta=0$ are replaced by an oscillating function which is typical for this kind of regularization. By using the Carstensen interpolant as regularization of the data, this can be avoided according to the local definition of this interpolation operator. But we note that these oscillations are a feature of the former regularization approach and do not disturb the approximation order.

\section{Extensions}

Remark 5.1. The paper is written for two-dimensional domains. However most results also hold in the three-dimensional case or can simply be extended to this one. The most crucial issue is the regularity which we have used for the corresponding adjoint problem since in three dimensional domains not only corner singularities but also edge singularities need to be taken into account.

Remark 5.2. We assume $f \in H^{-1}(\Omega)$ for the discretization error estimates of Corollary 3.3. This is only for simplicity. For defining the very weak solution and the numerical solution we only need $f \in\left(H_{0}^{1}(\Omega) \cap H_{\Delta}^{1}(\Omega)\right)^{\prime} \cap Y_{0 h}^{\prime}$ such that we could admit right hand sides from $L^{1}(\Omega)$ and Dirac measures as well. However, in this case the discretization error analysis demands an adapted proof, which exceeds the scope of this paper.

Remark 5.3. We assume $u \in L^{2}(\Gamma)$ for simplicity. The case $u \in H^{t}(\Gamma), t>0$, is also of interest in the analysis of Dirichlet control problems, see [1]. The results can be 


\begin{tabular}{crcccc}
\hline mesh size $h$ & $\#$ unknowns & $\left\|y-y_{h, 2}\right\|_{L^{2}\left(\Omega_{\omega}\right)}$ & eoc & $\left\|y-y_{h, C}\right\|_{L^{2}\left(\Omega_{\omega}\right)}$ & eoc \\
\hline 0.50000 & 19 & 0.26142 & & 0.26794 & \\
0.25000 & 61 & 0.18577 & 0.49289 & 0.18973 & 0.49794 \\
0.12500 & 217 & 0.13172 & 0.49600 & 0.13426 & 0.49899 \\
0.06250 & 817 & 0.09331 & 0.49745 & 0.09497 & 0.49940 \\
0.03125 & 3169 & 0.06605 & 0.49838 & 0.06717 & 0.49965 \\
0.01562 & 12481 & 0.04674 & 0.49902 & 0.04750 & 0.49982 \\
0.00781 & 49537 & 0.03306 & 0.49942 & 0.03359 & 0.49992 \\
0.00390 & 197377 & 0.02338 & 0.49967 & 0.02375 & 0.49998 \\
\hline expected & \multicolumn{5}{c}{0.5} \\
\hline
\end{tabular}

Table 1: Discretization errors and experimental orders of convergence (eoc) for $\omega=3 \pi / 4$. Expected convergence rate: $1 / 2$

\begin{tabular}{crcccc}
\hline mesh size $h$ & $\#$ unknowns & $\left\|y-y_{h, 2}\right\|_{L^{2}\left(\Omega_{\omega}\right)}$ & eoc & $\left\|y-y_{h, C}\right\|_{L^{2}\left(\Omega_{\omega}\right)}$ & eoc \\
\hline 0.50000 & 33 & 0.73622 & & 0.77007 & \\
0.25000 & 113 & 0.64484 & 0.19118 & 0.67086 & 0.19897 \\
0.12500 & 417 & 0.56841 & 0.18201 & 0.58915 & 0.18737 \\
0.06250 & 1601 & 0.50328 & 0.17555 & 0.52022 & 0.17950 \\
0.03125 & 6273 & 0.44674 & 0.17194 & 0.46091 & 0.17464 \\
0.01562 & 24833 & 0.39711 & 0.16987 & 0.40920 & 0.17166 \\
0.00781 & 98817 & 0.35330 & 0.16865 & 0.36376 & 0.16982 \\
0.00390 & 394241 & 0.31448 & 0.16793 & 0.32362 & 0.16868 \\
\hline expected & \multicolumn{5}{c}{0.16667} \\
\hline
\end{tabular}

Table 2: Discretization errors and experimental orders of convergence (eoc) for $\omega=3 \pi / 2$. Expected convergence rate: $1 / 6$

\begin{tabular}{crcccc}
\hline mesh size $h$ & \# unknowns & $\left\|y-y_{h, 2}\right\|_{L^{2}\left(\Omega_{\omega}\right)}$ & eoc & $\left\|y-y_{h, C}\right\|_{L^{2}\left(\Omega_{\omega}\right)}$ & eoc \\
\hline 0.50000 & 46 & 1.1049 & & 1.1141 & \\
0.25000 & 159 & 1.0693 & 0.04721 & 1.0732 & 0.05406 \\
0.12500 & 589 & 1.0491 & 0.02749 & 1.0513 & 0.02967 \\
0.06250 & 2265 & 1.0367 & 0.01715 & 1.0384 & 0.01782 \\
0.03125 & 8881 & 1.0281 & 0.01207 & 1.0296 & 0.01226 \\
0.01562 & 35169 & 1.0213 & 0.00956 & 1.0228 & 0.00962 \\
0.00781 & 139969 & 1.0154 & 0.00832 & 1.0169 & 0.00834 \\
0.00390 & 558465 & 1.0100 & 0.00771 & 1.0114 & 0.00772 \\
\hline expected & \multicolumn{5}{c}{0.00704} \\
\hline
\end{tabular}

Table 3: Discretization errors and experimental orders of convergence (eoc) for $\omega=$ $355 \pi / 180$, Expected convergence rate: $180 / 355-1 / 2=1 / 142 \approx 0.007$ 
improved in this more regular case: With $s$ from Corollary 3.3 we have

$$
\left\|y-y^{h}\right\|_{L^{2}(\Omega)} \leq c\left\|u-u^{h}\right\|_{H^{-s}(\Gamma)} \leq c h^{s+t}\|u\|_{H^{t}(\Gamma)}, \quad t \in\left[0, \frac{1}{2}\right] .
$$

The first step is again the application of Lemma 2.12, while the second can be proved in analogy to Lemma 2.13. The necessary prerequisites are already provided in Lemma A.2 and Remark A.3. Furthermore, when we check the proof of Lemma 3.2 we find that we obtain

$$
\left\|y_{0}^{h}-y_{0 h}\right\|_{L^{2}(\Omega)} \leq c h^{s}\left(h^{1 / 2}\|f\|_{H^{-1}(\Omega)}+h^{t}\left\|u^{h}\right\|_{H^{t}(\Gamma)}\right), \quad t \in\left[0, \frac{1}{2}\right] .
$$

Hence it remains to prove

$$
\left\|u^{h}\right\|_{H^{t}(\Gamma)} \leq c\|u\|_{H^{t}(\Gamma)},
$$

in order to conclude

$$
\left\|y-y_{h}\right\|_{L^{2}(\Omega)} \leq c h^{s+t}\left(h^{1 / 2-t}\|f\|_{H^{-1}(\Omega)}+\|u\|_{H^{t}(\Gamma)}\right), \quad t \in\left[0, \frac{1}{2}\right] .
$$

The estimate (5.1) is known for domains and can be proved for $t \in[0,1]$ also for the boundary $\Gamma$ by using the inverse inequality and the approximation properties of $u^{h}$ and the Ritz projection. To this end recall that $\Gamma_{j}, j=1, \ldots, N$, are the boundary segments of $\Gamma$, and let $P_{h}^{j}$ be the Ritz projection on $\left.Y_{h}^{\partial}\right|_{\Gamma_{j}}$. Then we have

$$
\begin{aligned}
\left\|u^{h}\right\|_{H^{1}(\Gamma)} & \leq \sum_{j=1}^{N}\left\|u^{h}-P_{h}^{j} u\right\|_{H^{1}\left(\Gamma_{j}\right)}+\sum_{j=1}^{N}\left\|P_{h}^{j} u\right\|_{H^{1}\left(\Gamma_{j}\right)} \\
& \leq c h^{-1} \sum_{j=1}^{N}\left\|u^{h}-P_{h}^{j} u\right\|_{L^{2}\left(\Gamma_{j}\right)}+\sum_{j=1}^{N}\|u\|_{H^{1}\left(\Gamma_{j}\right)} \\
& \leq c h^{-1}\left\|u-u^{h}\right\|_{L^{2}(\Gamma)}+c h^{-1} \sum_{j=1}^{N}\left\|u-P_{h}^{j} u\right\|_{L^{2}\left(\Gamma_{j}\right)}+\|u\|_{H^{1}(\Gamma)} \\
& \leq c\|u\|_{H^{1}(\Gamma)},
\end{aligned}
$$

i. e., we have (5.1) for $t=1$. Since we proved (5.1) for $t=0$ in Lemma 2.13 we get the desired result by interpolation in Sobolev spaces.

\section{Appendix: Error estimates for the Carstensen interpolant}

Recall from Subsection 2.5 that the piecewise linear Carstensen interpolant is defined via

$$
C_{h} u=\sum_{x \in \mathcal{N}_{\Gamma}} \pi_{x}(u) \lambda_{x}, \quad \pi_{x}(u)=\frac{\int_{\Gamma} u \lambda_{x}}{\int_{\Gamma} \lambda_{x}}=\frac{\left(u, \lambda_{x}\right)_{\Gamma}}{\left(1, \lambda_{x}\right)_{\Gamma}} .
$$

where $\lambda_{x}$ is the standard hat function related to $x$. 
Lemma A.1. The piecewise linear Carstensen interpolant satisfies the error estimate

$$
\left\|\varphi-C_{h} \varphi\right\|_{L^{2}(\Gamma)} \leq c h^{s}\|\varphi\|_{H^{s}(\Gamma)},
$$

for $\varphi \in H^{s}(\Gamma), s \in[0,1]$.

Proof. The interpolation error estimate (A.2) is in principle contained in [5], however, it is there an estimate on a domain such that we sketch the proof here.

For $s=0$ this estimate follows from the stability property

$$
\left\|\pi_{x}(u)\right\|_{L^{2}\left(\omega_{x}\right)} \leq c\|u\|_{L^{2}\left(\omega_{x}\right)},
$$

where $\omega_{x}$ is the support of $\lambda_{x}$ on $\Gamma$.

For $s=1$ we use that $\pi_{x}(w)=w$ for all constants $w$ such that

$$
\left\|u-\pi_{x}(u)\right\|_{L^{2}\left(\omega_{x}\right)}=\left\|(u-w)-\pi_{x}(u-w)\right\|_{L^{2}\left(\omega_{x}\right)} \leq c\|u-w\|_{L^{2}\left(\omega_{x}\right)} \leq c h\|u\|_{H^{1}\left(\omega_{x}\right)}
$$

via the Deny-Lions lemma, see also [10, Lemma 4.3] where the piecewise affine and Lipschitz continuous transformation of $\omega_{x}$ to some reference domain is discussed in detail. For a boundary edge with end points $x_{1}$ and $x_{2}$ we have

$$
\left\|u-C_{h} u\right\|_{L^{2}(e)}=\left\|\left(u-\pi_{x_{1}}(u)\right) \lambda_{x_{1}}+\left(u-\pi_{x_{2}}(u)\right) \lambda_{x_{2}}\right\|_{L^{2}(e)} \leq c \sum_{i=1}^{2}\left\|u-\pi_{x_{i}}(u)\right\|_{L^{2}(e)} .
$$

From these two estimates we obtain (A.2) in the case $s=1$. In the remaining case $s \in(0,1)$ the error estimate (A.2) follows by interpolation of Sobolev spaces.

Lemma A.2. The piecewise linear Carstensen interpolant satisfies the error estimate

$$
\left\|u-C_{h} u\right\|_{H^{-s}(\Gamma)} \leq c h^{s+t}\|u\|_{H^{t}(\Gamma)}, \quad s \in[0,1], \quad t \in[0,1],
$$

as well as the stability estimate

$$
\left\|C_{h} u\right\|_{L^{2}(\Gamma)} \leq c\|u\|_{L^{2}(\Gamma)} .
$$

Proof. The second estimate follows directly from the fact that $0 \leq \lambda_{x} \leq 1$ and the stability property (A.3).

The first estimate is in principle contained in [10], however, it is there an estimate on a domain such that we sketch the proof here. First we notice that the definition of $\pi_{x}(u)$ is equivalent to $\left(u-\pi_{x}(u), \lambda_{x}\right)_{\Gamma}=0$ and hence we have

$$
\left(u-\pi_{x}(u), \pi_{x}(\varphi) \lambda_{x}\right)_{\Gamma}=0 \quad \forall \varphi \in H^{s}(\Gamma) .
$$

With this identity and with $\sum_{x} \lambda_{x}=1$ we get

$$
\begin{aligned}
\left(u-C_{h} u, \varphi\right)_{\Gamma} & =\left(u \sum_{x} \lambda_{x}-\sum_{x} \pi_{x}(u) \lambda_{x}, \varphi\right)_{\Gamma}=\sum_{x}\left(u-\pi_{x}(u), \varphi \lambda_{x}\right)_{\Gamma} \\
& =\sum_{x}\left(u-\pi_{x}(u),\left(\varphi-\pi_{x}(\varphi)\right) \lambda_{x}\right)_{\Gamma} \\
& \leq \sum_{x}\left\|u-\pi_{x}(u)\right\|_{L^{2}\left(\omega_{x}\right)}\left\|\varphi-\pi_{x}(\varphi)\right\|_{L^{2}\left(\omega_{x}\right)}
\end{aligned}
$$


where $\omega_{x}$ is again the support of $\lambda_{x}$ on $\Gamma$. With similar arguments as in the proof of Lemma A.1 we conclude

$$
\left(u-C_{h} u, \varphi\right)_{\Gamma} \leq c h^{s+t}\|u\|_{H^{t}(\Gamma)}\|\varphi\|_{H^{s}(\Gamma)} .
$$

By the definition of the negative norm,

$$
\left\|u-C_{h} u\right\|_{H^{-s}(\Gamma)}=\sup _{\varphi \in H^{s}(\Gamma), \varphi \neq 0} \frac{\left(u-C_{h} u, \varphi\right)_{\Gamma}}{\|\varphi\|_{H^{s}(\Gamma)}},
$$

we obtain the assertion of the lemma.

Remark A.3. Note that this error estimate holds also for the $L^{2}$-projection,

$$
\left\|u-\Pi_{h} u\right\|_{H^{-s}(\Gamma)} \leq c h^{s+t}\|u\|_{H^{t}(\Gamma)}, \quad s \in[0,1], \quad t \in[0,1] .
$$

It can be proved similarly by using $\left(u-\Pi_{h} u, \varphi\right)_{\Gamma}=\left(u-\Pi_{h} u, \varphi-\Pi_{h} \varphi\right)_{\Gamma}$.

Acknowledgement The authors thank Markus Melenk and Christian Simader for helpful discussions.

\section{References}

[1] T. Apel, M. Mateos, J. Pfefferer, and A. Rösch. On the regularity of the solutions of Dirichlet optimal control problems in polygonal domains. Preprint arXiv:1505.00413, 2015.

[2] T. Apel, S. Nicaise, and J. Pfefferer. A dual singular complement method for the numerical solution of the Poisson equation with $L^{2}$ boundary data in non-convex domains. Preprint arXiv:1505.00414, 2015.

[3] I. Babuška. Error-bounds for finite element method. Numerische Mathematik, 16:322-333, 1970/1971.

[4] M. Berggren. Approximations of very weak solutions to boundary-value problems. SIAM J. Numer. Anal., 42(2):860-877, 2004.

[5] C. Carstensen. Quasi-interpolation and a posteriori error analysis in finite element methods. M2AN, Math. Model. Numer. Anal., 33(6):1187-1202, 1999.

[6] E. Casas and J.-P. Raymond. Error estimates for the numerical approximation of Dirichlet boundary control for semilinear elliptic equations. SIAM J. Control Optim., 45(5):1586-1611, 2006.

[7] J. Chabrowski. The Dirichlet problem with $L^{2}$-boundary data for elliptic linear equations. Number 1482 in Lecture Notes in Mathematics. Berlin etc.: SpringerVerlag, 1991. 
[8] P. G. Ciarlet. The finite element method for elliptic problems. North-Holland, Amsterdam, 1978. Reprinted by SIAM, Philadelphia, 2002.

[9] M. Costabel. Boundary integral operators on Lipschitz domains: elementary results. SIAM J. Math. Anal., 19(3):613-626, 1988.

[10] J. C. de los Reyes, C. Meyer, and B. Vexler. Finite element error analysis for stateconstrained optimal control of the Stokes equations. Control Cybernet., 37(2):251$284,2008$.

[11] K. Deckelnick, A. Günther, and M. Hinze. Finite element approximation of Dirichlet boundary control for elliptic PDEs on two- and three-dimensional curved domains. SIAM J. Control Optim., 48(4):2798-2819, 2009.

[12] D. A. French and J. T. King. Approximation of an elliptic control problem by the finite element method. Numer. Funct. Anal. Optimization, 12(3-4):299-314, 1991.

[13] P. Grisvard. Elliptic problems in nonsmooth domains, volume 24 of Monographs and Studies in Mathematics. Pitman, Boston-London-Melbourne, 1985.

[14] P. Grisvard. Singularities in boundary value problems, volume 22 of Research Notes in Applied Mathematics. Springer, New York, 1992.

[15] D. Jerison and C. E. Kenig. The inhomogeneous Dirichlet problem in Lipschitz domains. Journal of Functional Analysis, 130(1):161-219, 1995.

[16] J.-L. Lions and E. Magenes. Problèmes aux limites non homogènes et applications. Vol. 1, 2. Travaux et Recherches Mathématiques. Dunod, Paris, 1968.

[17] S. May, R. Rannacher, and B. Vexler. Error analysis for a finite element approximation of elliptic Dirichlet boundary control problems. SIAM J. Control Optim., 51(3):2585-2611, 2013.

[18] V. G. Maz'ya and B. Plamenevskiı̌. $L_{p}$-estimates of solutions of elliptic boundary value problems in domains with edges. Trudy Moskov. Mat. Obshch., 37:49-93, 1978. In Russian. Translated in Trans. Mosc. Math. Soc., 1:49-97, 1980.

[19] M. Mitrea and M. Taylor. Boundary layer methods for Lipschitz domains in Riemannian manifolds. J. Funct. Anal., 163(2):181-251, 1999.

[20] S. Nicaise. Polygonal Interface Problems, volume 39 of Methoden und Verfahren der mathematischen Physik. Peter Lang GmbH, Europäischer Verlag der Wissenschaften, Frankfurt/M., 1993.

[21] J. Pfefferer. Numerical analysis for elliptic Neumann boundary control problems on polygonal domains. $\mathrm{PhD}$ thesis, Universität der Bundeswehr München, 2014. http://athene. bibl. unibw-muenchen. de:8081/node?id=92055. 
[22] C. G. Simader. On Dirichlet's boundary value problem. An $L^{p}$-theory based on a generalization of Gärding's inequality. Lecture Notes in Mathematics, Vol. 268. Springer-Verlag, Berlin, 1972.

[23] C. G. Simader and H. Sohr. The Dirichlet problem for the Laplacian in bounded and unbounded domains. Harlow: Addison Wesley Longman, 1996.

[24] G. Verchota. Layer potentials and regularity for the Dirichlet problem for Laplace's equation in Lipschitz domains. Journal of Functional Analysis, 59(3):572-611, 1984. 